

Cite this: Phys. Chem. Chem. Phys., 2016, 18, 6580

Received 10th December 2015, Accepted 1st February 2016

DOI: $10.1039 / c 5 c p 07612 a$

www.rsc.org/pccp

\section{A general view on the reactivity of the oxygen-functionalized graphene basal plane}

\begin{abstract}
Ana S. Dobrota, ${ }^{a}$ Igor A. Pašti, ${ }^{a}$ Slavko V. Mentus ${ }^{a b}$ and Natalia V. Skorodumova ${ }^{c d}$
In this contribution we inspect the adsorption of $\mathrm{H}, \mathrm{OH}, \mathrm{Cl}$ and $\mathrm{Pt}$ on oxidized graphene using DFT calculations. The introduction of epoxy and hydroxyl groups on the graphene basal plane significantly alters its chemisorption properties, which can be attributed to the deformation of the basal plane and the type and distribution of these groups. We show that a general scaling relation exists between the hydrogen binding energies and the binding energies of other investigated adsorbates, which allows for a simple probing of the reactivity of oxidized graphene with only one adsorbate. The electronic states of carbon atoms located within the $2 \mathrm{eV}$ interval below the Fermi level are found to be responsible for the interaction of the basal plane with the chosen adsorbates. The number of electronic states situated in this energy interval is shown to correlate with hydrogen binding energies.
\end{abstract}

\section{Introduction}

Graphene is a single layer of carbon atoms arranged in a twodimensional (2D) honeycomb lattice. Although formerly known as a purely 'academic' toy-model material, this zero-gap semiconductor is now at the center of advanced technologies due to its extraordinary properties. ${ }^{1}$ Pure graphene has high mechanical stiffness, good thermal conductivity and exceptional charge carrier mobility. ${ }^{2}$ Based on these properties, graphene has found applications in many fields, such as electronics, ${ }^{3}$ gas sensing ${ }^{4}$ and energy storage. ${ }^{5,6}$ However, graphene is chemically inert and difficult to functionalize ${ }^{7}$ which limits its application beyond the fields relying on its intrinsic properties. On the other hand, graphene is usually produced by the reduction of graphene oxide (GO), which contains a large amount of oxygen. ${ }^{8}$ On the path from GO to graphene one can find oxygen-functionalized graphene with a variable $\mathrm{C} / \mathrm{O}$ ratio displaying new physical and chemical properties. By a controllable reduction of GO it is possible to tune the $\mathrm{C} / \mathrm{O}$ ratio and conductivity to optimize the material for targeted applications. ${ }^{9}$ Oxygen functional groups (epoxy, hydroxyl, carbonyl and carboxyl ${ }^{10}$ ) induce $\mathrm{sp}^{3}$ defects in the graphene structure and distort the $\pi$ conjugated system, reducing the strength and conductivity of the material. ${ }^{11}$ However, these

\footnotetext{
${ }^{a}$ University of Belgrade, Faculty of Physical Chemistry, Studentski trg 12-16, 11158 Belgrade, Serbia. E-mail: igor@ffh.bg.ac.rs; Fax: +381 112187 133; Tel: +381113336 628

${ }^{b}$ Serbian Academy of Sciences and Arts, Knez Mihajlova 35, 11000 Belgrade, Serbia

${ }^{c}$ Department of Physics and Astronomy, Uppsala University, Box 516, 75120 Uppsala, Sweden

${ }^{d}$ Department of Materials Science and Engineering, School of Industrial Engineering and Management, KTH - Royal Institute of Technology, Brinellvägen 23,

10044 Stockholm, Sweden
}

functional groups also open up new application possibilities. For example, the presence of oxygen functional groups boosts charge storage $^{12}$ and metal ion storage capacity ${ }^{13}$ of graphene. Also, GO and reduced GO can be used as integral parts of composite materials for various applications. ${ }^{14,15}$

Epoxy and hydroxyl groups prefer to be located on the graphene basal plane rather than at its edges, ${ }^{16}$ causing the corrugation of the graphene sheet. ${ }^{9}$ The presence of oxygen surface groups alters the electronic structure of graphene and at high coverage can lead to band gap opening and conductivity reduction of the functionalized graphene. ${ }^{17}$ However, these groups can also induce other specific properties lacking in the case of pristine graphene (p-graphene). For example, oxygen groups can interact strongly with $\mathrm{Li}^{+}$ions. ${ }^{13}$ Due to the presence of functional groups, an $\mathrm{sp}^{2} \rightarrow \mathrm{sp}^{3}$ re-hybridization takes place to the extent which depends on the $\mathrm{C} / \mathrm{O}$ ratio. Considering a partial re-hybridization of the $\mathrm{C}$ states, one can anticipate the formation of surface dangling bonds, which can alter the reactivity of the basal plane itself.

In this contribution we investigate the reactivity of the basal plane of oxygen-functionalized graphene using Density Functional Theory (DFT) calculations. In particular, we analyze the adsorption of four technologically important species $(\mathrm{H}, \mathrm{OH}, \mathrm{Cl}$, and $\mathrm{Pt})$ on epoxy- and hydroxyl-functionalized graphene with a $\mathrm{C} / \mathrm{O}$ ratio of $8 / 1$ and search for similarities, differences and universalities in their interaction with the basal plane of the oxidized graphene. The primary aim of this work is to establish a link between the electronic structure of the oxidized graphene and its chemisorption properties. Such relationships are known for transition metal surfaces ${ }^{18-20}$ but are not yet established for graphene. However, it would be very important to have them for all types of surfaces as they allow us to better understand the reactivity of solid surfaces and to propose ways to advance their properties. 


\section{Computational details}

The first principles DFT calculations were carried out within the generalized gradient approximation (GGA) and PerdewBurke-Ernzerhof (PBE) exchange-correlation functional ${ }^{21}$ using ultrasoft pseudopotentials as implemented in the PWscf code of the Quantum ESPRESSO distribution. ${ }^{22}$ The Kohn-Sham orbitals were expanded in a plane wave basis set with a kinetic energy cutoff of $30 \mathrm{Ry}$, while the charge density cutoff was 448 Ry. Pristine graphene was modeled as a 32 carbon atom layer within an orthorhombic $9.88 \times 8.65 \times 16 \AA$ supercell, i.e. a $p(4 \times 4)$ structure in the $x y$ plane, similar to that in ref. 23 . Oxidized graphene (ox-graphene) was modeled as p-graphene with oxygen groups attached to both sides of the layer. Both epoxy- and hydroxyl-functionalized graphene were considered. For the former case we considered two possible distributions of epoxy-groups on the graphene basal plane (models named epoxygraphene-1 and epoxy-graphene-2). The detailed description of the surface models is given in ref. 24. The first irreducible Brillouin zone was sampled using a $\Gamma$-centered $3 \times 3 \times 1$ grid of $k$-points generated by the general Monkhorst-Pack scheme. ${ }^{25}$ The convergence with respect to the vacuum layer thickness and the $k$-point mesh was confirmed. For the electronic structure analysis a denser, $9 \times 11 \times 1 k$-point grid was used. Atomic positions were fully relaxed until the residual forces acting on atoms were smaller than $0.005 \mathrm{eV} \AA^{-1}$. The charge redistribution was analyzed using the Bader algorithm ${ }^{26}$ on a charge density grid by Henkelman et $a l^{27}$

The interaction of chosen adsorbates with the p-graphene and ox-graphene surfaces was quantified in terms of binding energies $\left(E_{\mathrm{b}}\right)$, defined as

$$
E_{\mathrm{b}}(\mathrm{X})=E_{\text {subs }+\mathrm{X}}-\left(E_{\text {subs }}+E_{\mathrm{X}}\right)
$$

where $E_{\text {subs+X }}, E_{\text {subs }}$ and $E_{\mathrm{X}}$ denote the total energy of the substrate with adsorbed $\mathrm{X}(\mathrm{X}=\mathrm{H}, \mathrm{OH}, \mathrm{Cl}$ or $\mathrm{Pt})$, the total energy of the bare substrate and the total energy of the isolated adsorbate X. $E_{\mathrm{X}}$ was obtained by spin-polarized calculations. Note that the molecular dissociation of $\mathrm{H}_{2}$ and $\mathrm{Cl}_{2}$ is not included in $E_{\mathrm{b}}(\mathrm{X})$. We used the results of non-spin polarized calculations to map the reactivity of the ox-graphene basal planes, but tested the spin polarization effects explicitly for a number of systems investigated here. The ox-graphene models we used were found to be nonmagnetic.

\section{Results and discussion}

\subsection{Adsorption on p-graphene}

We observed that $\mathrm{H}, \mathrm{OH}$ and $\mathrm{Cl}$ prefer the C-top adsorption sites, whereas Pt prefers the two-fold $\mathrm{C}-\mathrm{C}$ bridge site on p-graphene. The calculated binding energies and bond lengths, listed in Table 1, are in good agreement with the previous results found in the literature. The effect of spin polarization on the calculated binding energies is minor (up to $0.05 \mathrm{eV}$, Table 1) and this trend also holds for the adsorption on the ox-graphene surfaces.

Once we set the benchmarks for the reactivity of the p-graphene basal plane we proceed to the adsorption on the O-functionalized graphene.
Table 1 Adsorption parameters for the adsorption of $\mathrm{H}, \mathrm{OH}, \mathrm{Cl}$ and $\mathrm{Pt}$ on $\mathrm{p}$-graphene: preferred adsorption sites, carbon-adsorbate bond lengths $(d(C-X))$, binding energies calculated within both non-spin polarized $\left(E_{\mathrm{b}}^{\text {no spin }}(\mathrm{X})\right)$ and spin polarized formalism $\left(E_{\mathrm{b}}^{\text {spin }}(\mathrm{X})\right)$. For comparison, corresponding binding energies found in the literature $\left(E_{\mathrm{b}}^{\text {literature }}(\mathrm{X})\right)$ are listed in the last column

\begin{tabular}{llllll}
\hline Adsorbate & $\begin{array}{l}\text { Adsorption } \\
\text { site }\end{array}$ & $d(\mathrm{X}-\mathrm{C}) / \mathrm{A} \AA \begin{array}{l}E_{\mathrm{b}}^{\text {no spin }} \\
\mathrm{eV}\end{array}$ & $\begin{array}{l}E_{\mathrm{b}}^{\text {spin }}(\mathrm{X}) / \\
\mathrm{eV}\end{array}$ & $\begin{array}{l}E_{\mathrm{b}}^{\text {literature }} \\
\mathrm{eV}\end{array}$ \\
\hline $\mathrm{OH}$ & C-top & 1.51 & -0.69 & -0.70 & $\begin{array}{l}-0.21^{c} \\
-0.54^{d}\end{array}$ \\
& & & & & $-0.70^{e}$ \\
& & & & & $-0.79^{a}$ \\
$\mathrm{H}$ & C-top & 1.13 & -0.84 & -0.89 & $-0.89^{b}$ \\
& & & & & $-0.80^{f}$ \\
$\mathrm{Cl}$ & C-top & 3.23 & -0.94 & -0.98 & $-1.13^{g}$ \\
& & & & & $-1.55^{h}$ \\
$\mathrm{Pt}$ & C-C bridge & 2.11 & -1.56 & -1.57 & $-1.57^{i}$ \\
& & & & & $-2.04^{j}$
\end{tabular}

${ }^{a}$ Ref. 28. ${ }^{b}$ Ref. $29{ }^{c}$ Ref. $30{ }^{d}$ Ref. $31 .^{e}$ Ref. $32 .{ }^{f}$ Ref. 33. ${ }^{g}$ Ref. 34. ${ }^{h}$ Ref. 23. ${ }^{i}$ Ref. $35 .{ }^{j}$ Ref. 36.

\subsection{Adsorption on O-functionalized graphene: an overview}

To analyze the adsorption of $\mathrm{H}, \mathrm{OH}, \mathrm{Cl}$ and $\mathrm{Pt}$ on oxidized graphene we considered several $\mathrm{C}$ atoms in the basal plane as possible adsorption sites, chosen by their electronic structure and the symmetry of the simulation cell. The models of oxidized graphene used here are discussed in detail in ref. 24. It should be noted that we do not aim to find the lowest possible configuration of oxygen groups over the graphene basal plane, as discussed in ref. 37. We use these groups to disturb the electronic structure of $\mathrm{C}$ sites and their local arrangement in order to obtain the link between the binding energies of different adsorbates and the binding energies and the local electronic structure. In all the cases we observe a significant modification of the adsorption properties of the graphene basal plane upon the introduction of oxygen functional groups (Fig. 1). Although some previous reports suggested that the chemical reaction between an adsorbate and an oxygen functional group could occur, ${ }^{38}$ the only effect we have seen in some cases, discussed below, is the movement of an adsorbate from the initial adsorption site while no by-product formation was observed.

As a rule, we observed an enhanced $\mathrm{H}$ adsorption on ox-graphene compared to that on p-graphene. The strongest $\mathrm{H}$ adsorption, with the energy of $-2.20 \mathrm{eV}$, is obtained for the hydroxylgraphene model. $\mathrm{H}$ adsorption is the weakest for the epoxygraphene-1 model, but it is still approx. $1 \mathrm{eV}$ stronger compared to that on p-graphene. Comparing different adsorption sites for each model, the strongest $\mathrm{H}$ binding was found at the $\mathrm{C}$ sites adjacent to the O-functional groups. This is the trend for all the three ox-graphene models. These results suggest that the introduction of the oxygen functional groups enhances $\mathrm{H}$ adsorption on the graphene basal plane in such a way that it binds $\mathrm{H}$ as strongly as coinage metal surfaces. ${ }^{40}$

This also indicates a possibility of using O-functionalized graphene in the field of hydrogen storage as the enhanced $\mathrm{H}$ binding points to higher storage capacity. Graphene-based materials 


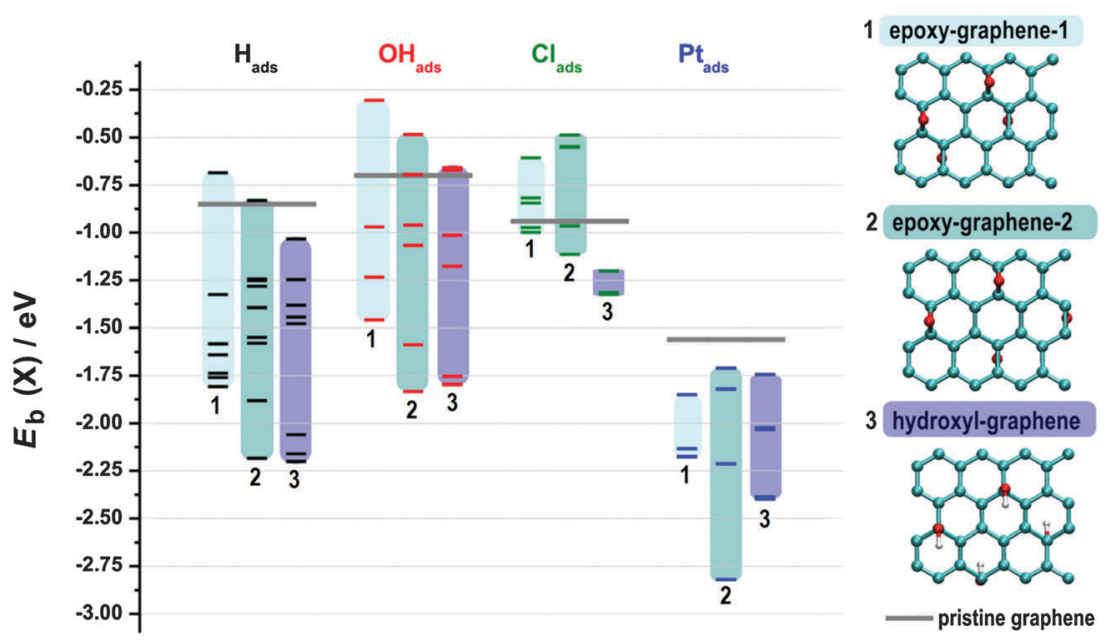

Fig. $1 \mathrm{H}, \mathrm{OH}, \mathrm{Cl}$ and Pt binding energies at different sites of the three ox-graphene models, calculated within non-spin polarized formalism. Columns marked with 1, 2 and 3 stand for $X$ binding energies on epoxy-graphene-1, epoxy-graphene-2 and hydroxyl-graphene models, respectively. Corresponding binding energies on the pristine graphene model are indicated by thicker, grey horizontal lines. The models of the oxidized graphene used in this work are given on the right. Graphical presentations were made using the VMD code. ${ }^{39}$

have indeed found applications in this field and are in the focus of further research. ${ }^{41}$

For the case of $\mathrm{OH}$ adsorption, which among the investigated adsorbates is the weakest on p-graphene, we see significant adsorption enhancement as compared to p-graphene, with $E_{\mathrm{b}}(\mathrm{OH})$ amounting to $-1.46 \mathrm{eV},-1.83 \mathrm{eV}$ and $-1.80 \mathrm{eV}$ for epoxygraphene-1, epoxy-graphene-2 and hydroxyl-graphene, respectively (Fig. 1). Although there are adsorption sites on ox-graphene, which bind $\mathrm{OH}$ more weakly than p-graphene, most of the $\mathrm{C}$ atoms bind $\mathrm{OH}$ much more strongly. The strongest adsorption is on the $\mathrm{C}$ atoms, which are the first neighbors to the $\mathrm{C}$ atoms binding the $\mathrm{O}$-functional groups, when $\mathrm{OH}$ adsorbs at the ortho position with respect to the O-functional groups on the surface. These observations match the results of Ghaderi and Peressi ${ }^{31}$ who have found a significant energy gain when different $\mathrm{O}$ functional groups (epoxy or hydroxyl) are adjacent to each other on the opposite sides of the graphene plane.

Halogenated graphene (fluoro- and chloro-graphene) is rather interesting in contemporary technologies as the tailoring of the electronic properties of graphene can be achieved through controlled halogenation. ${ }^{42,43}$ In our calculations the case of $\mathrm{Cl}$ adsorption is found to be somewhat different compared to the $\mathrm{H}$ and $\mathrm{OH}$ cases. $\mathrm{A} \mathrm{Cl}$ atom attaches to the C-top site of the epoxy-graphene model surfaces, and the bonding is slightly enhanced at the $\mathrm{C}$ sites which are the closest to the oxygen functional groups (Fig. 1). The enhancement is rather small and amounts to $0.13 \mathrm{eV}$ for the epoxy-graphene-2 model (Fig. 1). In fact, most of the $\mathrm{C}$ sites of the basal planes of the epoxygraphene bind $\mathrm{Cl}$ more weakly than those of p-graphene. This is likely due to the high electronegativity of $\mathrm{Cl}$ and electron deficiency of the basal plane of ox-graphene. When attached to p-graphene, $\mathrm{Cl}$ withdraws $0.47 \mathrm{e}$, but ox-graphene is electron deficient as the $\mathrm{O}$ functional groups have already drawn charge from the $\mathrm{C}$ basal plane. ${ }^{24}$ As a rule, we observe that when adsorbed at the site of the weakest bonding, $\mathrm{Cl}$ takes only up to
0.03 e less from epoxy-graphene than from p-graphene. However, at the strongly binding sites of epoxy-graphene models $\mathrm{Cl}$ takes up to 0.27 e less compared to p-graphene. In this case the $\mathrm{C}-\mathrm{Cl}$ bond is up to $41 \%$ shorter compared to the one on p-graphene. This clearly suggests that the contribution of covalent bonding to the unsaturated surface bond formed upon the functionalization of p-graphene is increased. Furthermore, in the majority of cases we observe an additional interaction between negatively charged $\mathrm{Cl}_{\mathrm{ads}}$ and positively charged $\mathrm{H}$ of the $\mathrm{OH}$ functional group on the hydroxyl-graphene model. This interaction is electrostatic in nature while some contribution by the hydrogen bond can also be expected. This additional attractive interaction is of long range nature and, when combined with the high mobility of $\mathrm{Cl}_{\mathrm{ads}}$ on the graphene basal plane, ${ }^{42}$ results in a stable configuration to which a large number of different starting adsorption geometries converged in our calculations. The contribution of this interaction to $E_{\mathrm{b}}(\mathrm{Cl})$ cannot be appropriately estimated but the instability of $\mathrm{Cl}$ adsorption at the basal plane sites of hydroxyl-graphene suggests reduced affinity of the basal plane for $\mathrm{Cl}$ as compared to that for p-graphene. The obtained results suggest that the oxidation of graphene might induce problems when it comes to the functionalization of graphene by $\mathrm{Cl}$. Also, one might say that controlling the graphene oxidation level can be useful for tuning the affinity of graphene for $\mathrm{Cl}$ so that desirable amounts of $\mathrm{Cl}$ can be loaded onto the graphene basal plane.

In the case of platinum adsorption on ox-graphene models we observe a decrease of binding energy for all the three models compared to that on p-graphene (Fig. 1), suggesting that the affinity of the basal plane for Pt is enhanced by the presence of oxygen functional groups. This effect has previously been reported in the literature for the case of O-containing graphene where the model of O-saturated vacancy was considered. ${ }^{23}$ Such a behavior is of tremendous importance when it comes to the production of highly dispersed and stable supported Pt-based catalysts. ${ }^{44,45}$ While Pt prefers a bridge site on p-graphene, here we observe 
that in some cases Pt moves to the C-top sites, which indicates its direct interaction with the surface dangling bonds. Again, the $\mathrm{C}$ sites closest to the functional groups are the strongest binding sites for $\mathrm{Pt}_{\mathrm{ads}}$, suggesting that these sites could be the place for the initial nucleation of Pt nanoparticles during the deposition of Pt onto oxidized graphene. Also, in some cases Pt adsorption is augmented by the $\mathrm{O}$ functional group as described in ref. 46 but the interaction with the surface $\mathrm{C}$ atoms still largely remains. As in the case of $\mathrm{Cl}$ adsorption, some of the considered sites of the $\mathrm{C}$ basal plane were found to be unstable for Pt adsorption, as the Pt adatom migrated to the adjacent site where stable adsorption could be achieved. As a rule, the unstable sites mostly include the weakly bonding ones also for other adsorbates $(\mathrm{OH}, \mathrm{H}, \mathrm{Cl})$.

The question is whether there are some universal relationships between the binding energies of different adsorbates. Revealing such a relationship could provide a simple tool for the assessment of surface reactivity towards various adsorbates using measurements with a single one. Such relations were observed for transition metal surfaces. ${ }^{47}$ To check this possibility, we correlated the binding energies of $\mathrm{OH}, \mathrm{Cl}$ and $\mathrm{Pt}$ at the stable adsorption sites to the $\mathrm{H}$ binding energy at the same adsorption sites. For the cases of Pt adsorption at two-fold sites we used the average of $\mathrm{H}$ binding energies on the two $\mathrm{C}$ atoms forming that bridge site. We observed excellent correlations, with the coefficient of determination $\left(R^{2}\right)$ higher than 0.99 and relative slope errors below $4 \%$ in all three cases (Fig. 2). This suggests a simple rule of thumb regarding the adsorption of simple adsorbates on oxidized graphene: the stronger the $\mathrm{H}$ adsorption at a given site is, the stronger the adsorption of other adsorbates at this site will be, as well. The scaling relations we provide have physically reasonable properties: they all have zero intercept. Colloquially said, if hydrogen adsorption is not present then the given adsorption site will be inert for the adsorption of other species as well. Naturally, some scattering of the data points is observed and could be related to the effect of local geometries and the interaction of the adsorbate with the $\mathrm{O}$ functional groups of ox-graphene. In the case of Pt this is also related to different types of adsorption sites for $\mathrm{Pt}_{\mathrm{ads}}$ and $\mathrm{H}_{\mathrm{ads}}$ (bridge $v s$. top).

The question is whether p-graphene also falls onto these lines. In the case of $\mathrm{OH}$ adsorption, the agreement is perfect (estimated $\mathrm{OH}$ binding energy is $-0.69 \mathrm{eV}$ ). In the case of Pt adsorption the binding energy is underestimated by $0.44 \mathrm{eV}$. However, the largest error is seen in the case of $\mathrm{Cl}$ adsorption. Nevertheless, we believe that this is due to the fact that the nature of bonding of the investigated adsorbates is different on p-graphene and ox-graphene, which is also reflected in the change of the Pt adsorption site, as previously discussed. The observed scaling relations must have their origin in the electronic structure of the ox-graphene, so in the next section we turn to this issue.

\subsection{Adsorption on O-functionalized graphene: the electronic structure insights}

Upon the inspection of the projected densities of states (PDOS) of ox-graphene with the considered adsorbates, one can note a strong hybridization between the $\mathrm{p}$ states of the binding $\mathrm{C}$ atoms and the adsorbate valence orbitals (Fig. 3). The states of

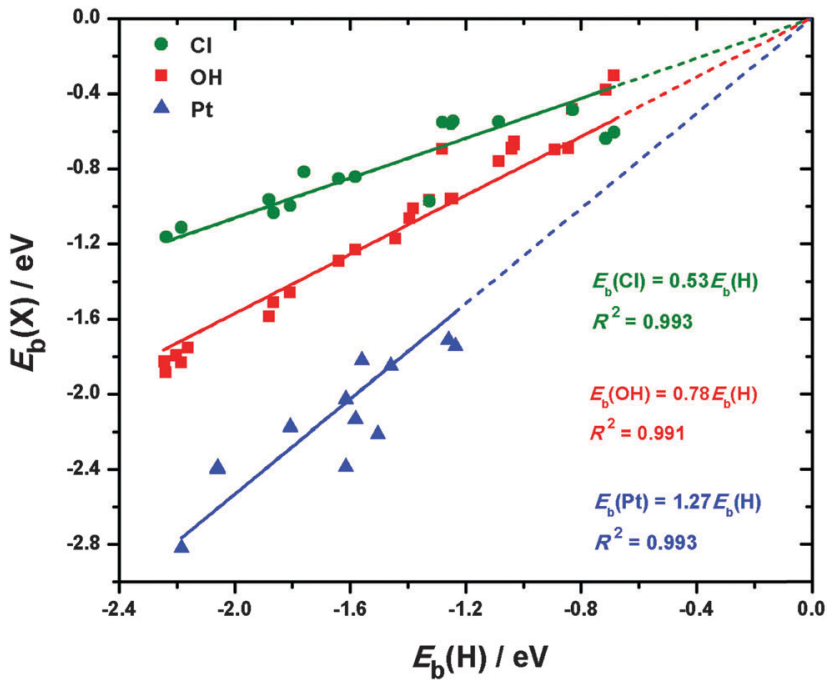

Fig. 2 Correlation between the $\mathrm{H}$ binding energy and the binding energies of other investigated adsorbates, taking into account all three ox-graphene models. Regression lines and coefficients of determination are given. For the case of $\mathrm{Cl}$ adsorption we excluded the data points where $\mathrm{Cl}$ interacts with the $\mathrm{H}$ atom of the $\mathrm{OH}$ group of hydroxyl-graphene.

the binding $\mathrm{C}$ atom and the adsorbates are well aligned and the adsorbate states are located in the upper parts of the ox-graphene valence band, close to the Fermi level $\left(E_{\mathrm{F}}\right.$; we set it to $0 \mathrm{eV}$ from hereafter) (Fig. 3).

The inspection of PDOS of hydrogen and carbon atoms for the strongest binding sites on all the three ox-graphene models (Fig. 3) displays a strong chemical interaction between the $\mathrm{H}$ and $\mathrm{C}$ valence states. Without going into details for each particular case, we notice that the carbon p state depletion in the range $-2 \mathrm{eV}$ to $0 \mathrm{eV}$ taking place upon adsorption is a general feature for all the studied systems (Fig. 3). This is a clear indication that precisely these states take an active role in the bonding of the graphene basal plane and all the investigated adsorbates, which are rather different in chemical terms.

The source of the enhanced reactivity of the basal plane $\mathrm{C}$ atoms must lie in the electronic structure of the bare ox-surface (i.e. without the adsorbate). A deeper look into the electronic structure of the ox-graphene models reveals that the $\mathrm{p}$ states of the $\mathrm{C}$ atoms which bind $\mathrm{H}$ more strongly show a higher number of electronic states very close to $E_{\mathrm{F}}$, while the s states show a more pronounced overlap with the $\mathrm{p}$ states at higher energies, suggesting higher $\mathrm{sp}^{2} \rightarrow \mathrm{sp}^{3}$ re-hybridization.

To visualize these states we performed the analysis of the Integrated Local Density of States (ILDOS) in the range $-2 \mathrm{eV}$ to $0 \mathrm{eV}$, which clearly showed that the $\mathrm{C}$ atoms which bind $\mathrm{H}$ more strongly possess a higher charge density in the specified energy window (Fig. 4, upper left panel). This means that upon the corrugation of the basal plane and electron redistribution due to the presence of oxygen functional groups the electronic states of some of the C atoms get "prepared" for bonding that ensures higher reactivity of oxidized graphene towards $\mathrm{H}$ compared to that of the p-graphene basal plane. As we have shown the binding energies of $\mathrm{OH}, \mathrm{Pt}$ and $\mathrm{Cl}$ scale with respect to $E_{\mathrm{b}}(\mathrm{H})$ 

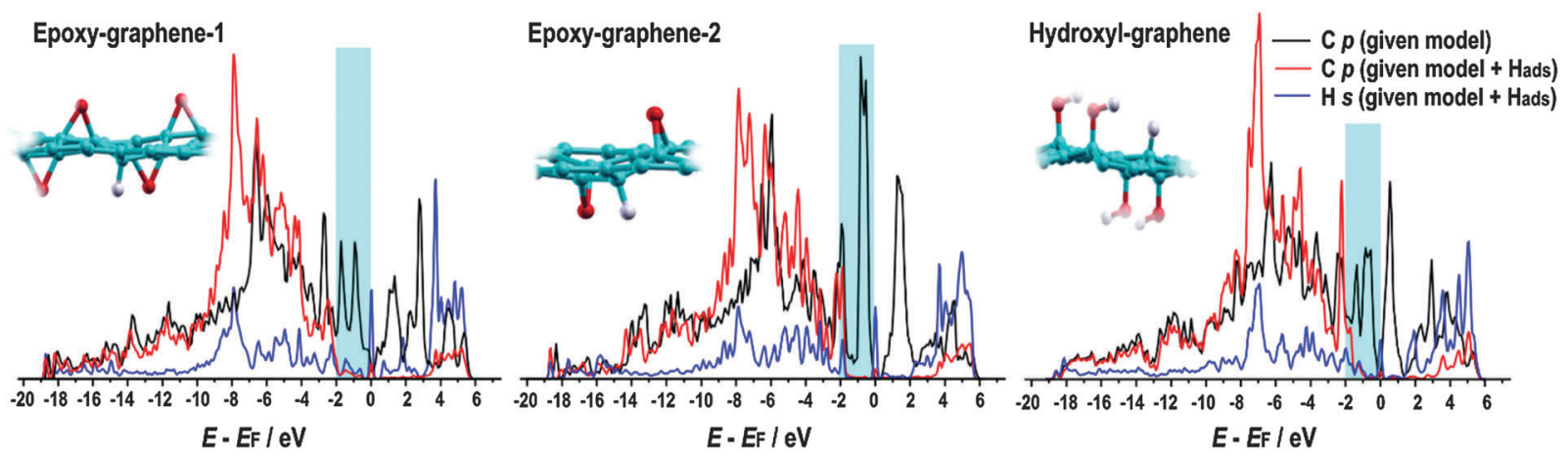

Fig. 3 A comparison of electronic structures of bare ox-graphene models (no adsorbate), and the most stable structures with $\mathrm{H}_{\mathrm{ads}}$, considering $\mathrm{H}_{\mathrm{ads}}$ and the binding $\mathrm{C}$ atom. Corresponding optimized structures, presented using the XCrySDen code, ${ }^{48}$ are provided as insets.

(Fig. 2) as well as with respect to each other. Obviously, the origin of the enhanced reactivity should be found in the electronic structure of the basal plane $\mathrm{C}$ atoms. The question is whether we can find a single electronic structure-based descriptor capable of predicting the adsorption trends on these types of surfaces. For transition metal surfaces such a descriptor, the d-band center $\left(E_{\mathrm{d} \text {-band }}\right)$, has been identified by Hammer and Nørskov. ${ }^{18,19}$ Based on the presented results, the number of states in the specified energy window right below $E_{\mathrm{F}}$ seems to be a logical choice. The correlation between the $\mathrm{H}$ binding energy and the integrated number of states obtained for the four selected $\mathrm{C}$ atoms of the epoxy-graphene- 1 model is shown in Fig. 4 (left).

Obviously, the correlation is not perfectly linear; however, at this point there is no reason to expect it should be like that, especially having in mind that the binding energy can be affected by the interaction between the adsorbate and the oxygen functional group. A rather good trend is observed indicating that the number of states located very close to the Fermi level determines the reactivity of a given $\mathrm{C}$ atom. The same correlation for all the three ox-graphene models and p-graphene is given in Fig. 4 (right). The scattering of the points is obvious but the overall trend is unambiguous. Of course, the choice of the energy widow or the integration of PDOS is, to a certain extent, arbitrary but it should include the states participating in the bonding. In fact, one can play with the integration window to maximize the correlation. Nonetheless, the main conclusion remains: the strength of the interaction between the basal plane of (modified) graphene and a given adsorbate is determined by the number of states just below the Fermi level. As an additional point, one should observe how sensitive $E_{\mathrm{b}}(\mathrm{H})$ is to the number of states below the Fermi level (Fig. 4, right) - the variation in the number of states of approx. $0.35 \mathrm{e}$ corresponds to the binding energy change of almost $1.5 \mathrm{eV}$.

The final electronic structure and the charge state of a given $\mathrm{C}$ atom ultimately depend on the distribution and the type of oxygen functional groups. ${ }^{24}$ To separate the effect of geometrical distortion of the graphene basal plane from electron draining by the oxygen functional groups we performed calculations for the hypothetical structures of the corrugated graphene obtained by the removal of the oxygen functional groups from the optimized models


Fig. 4 Left: Correlation between $\mathrm{H}$ binding energy on the selected sites of epoxy-graphene-1 model (as indicated in the inset, where 1 is the strongest binding site for $\mathrm{H}$ adsorption, and 4 the weakest) and the number of states between $-2 \mathrm{eV}$ and $E_{\mathrm{F}}$ (set to $0 \mathrm{eV}$ ). The upper left panel gives the Integrated Local Density of States in the same energy window for the analyzed $C$ atoms. Right: Correlation between $E_{\mathrm{b}}(\mathrm{H})$ and the number of states found between $-2 \mathrm{eV}$ and $E_{\mathrm{F}}$ for all three ox-graphene models. 
of ox-graphene. For the case of $\mathrm{H}$ adsorption all the binding energies were found to be between $-1.51 \mathrm{eV}$ and $-1.99 \mathrm{eV}$ while the charge transfer was almost unaffected by the removal of the $\mathrm{O}$ functional groups. Somewhat weaker adsorption upon the removal of the $\mathrm{O}$ functional groups was observed only for the strongest binding sites adjacent to the $\mathrm{O}$ functional groups in the O-containing models. This suggests that the dominant source of the enhanced reactivity of the graphene basal plane is, in fact, the corrugation of the structure. Reactivity is additionally tuned by the charge redistribution induced by the $\mathrm{O}$ functional groups. This conclusion is in line with the results of Tozzini and Pellegrini $^{49}$ who have tuned H-graphene binding just by the out-of-plane deformation of graphene without introducing any surface functional groups. This view is also supported by our calculations for the case of $\mathrm{Cl}$ adsorption. For the strongest binding sites we observed the additional strengthening of the $\mathrm{C}-\mathrm{Cl}$ bond by approx. $0.3 \mathrm{eV}$ while more charge is transferred to $\mathrm{Cl}_{\text {ads }}$. This is because the considered artificial structures have more electrons in the basal plane (i.e. the same amount as p-graphene) and also due to the appearance of dangling bonds induced by the deformation of the basal plane.

\section{Conclusions}

We have analyzed the adsorption of $\mathrm{H}, \mathrm{OH}, \mathrm{Cl}$ and $\mathrm{Pt}$ on three different models of graphene functionalized by epoxy and hydroxyl groups with the intention to establish a general view on the reactivity of the basal plane of the O-functionalized graphene. The basal plane of oxidized graphene shows enhanced chemisorption properties towards $\mathrm{H}, \mathrm{OH}$ and $\mathrm{Pt}$, while its affinity for $\mathrm{Cl}$ is generally decreased. We conclude that reactivity increases due to the deformation of the basal plane, resulting in the formation of surface dangling bonds. Reactivity is further tuned by specific oxygen functional groups. Clear general scaling relations between the binding energies of the investigated adsorbates have been revealed, which opens up a possibility of using a single probe adsorbate to obtain a general picture of the reactivity of the ox-graphene basal plane. The electronic states of carbon atoms located just below the Fermi level have been identified as responsible for the formation of chemical bonds with the investigated adsorbates. We have confirmed that the binding energy of hydrogen atoms scales with the number of states found in the energy window located between -2 and $0 \mathrm{eV} v s . E_{\mathrm{F}}$, indicating a general rule that the adsorption will be stronger if there are more electronic states close to the Fermi level.

\section{Acknowledgements}

This work was supported by the Swedish Research Links initiative of the Swedish Research Council (348-2012-6196). Computational resources are provided by the Swedish National Infrastructure for Computing (SNIC). A. S. D, I. A. P and S. V. M. acknowledge the support provided by the Serbian Ministry of Educations, Science and Technological Development through the project III45014.

\section{References}

1 A. K. Geim and K. S. Novoselov, The rise of graphene, Nature Mater., 2007, 6(3), 183-191.

2 M. Orlita, C. Faugeras, P. Plochocka, P. Neugebauer, G. Martinez, D. K. Maude, A.-L. Barra, M. Sprinkle, C. Berger, W. A. de Heer and M. Potemski, Approaching the Dirac point in highmobility multilayer epitaxial graphene, Phys. Rev. Lett., 2008, 101(26), 267601.

3 J. U. Park, S. Nam, M. S. Lee and C. M. Lieber, Synthesis of monolithic graphene-graphite integrated electronics, Nature Mater., 2012, 11(2), 120-125.

4 J. D. Fowler, M. J. Allen, V. C. Tung, Y. Yang, R. B. Kaner and B. H. Weiller, Practical chemical sensors from chemically derived graphene, ACS Nano, 2009, 3(2), 301-306.

5 M. D. Stoller, S. Park, Y. Zhu, J. An and R. S. Ruoff, Graphenebased ultracapacitors, Nano Lett., 2008, 8(10), 3498-3502.

6 C. Wang, D. Li, C. O. Too and G. G. Wallace, Electrochemical properties of graphene paper electrodes used in lithium batteries, Chem. Mater., 2009, 21(13), 2604-2606.

7 V. Georgakilas, M. Otyepka, A. B. Bourlinos, V. Chandra, N. Kim, K. C. Kemp, P. Hobza, R. Zboril and K. S. Kim, Functionalization of graphene: covalent and non-covalent approaches, derivatives and applications, Chem. Rev., 2012, 112(11), 6156-6214.

8 P. V. Kumar, N. M. Bardhan, S. Tongay, J. Wu, A. M. Belcher and J. C. Grossman, Scalable enhancement of graphene oxide properties by thermally driven phase transformation, Nature Chem., 2014, 6(2), 151-158.

9 K. A. Mkhoyan, A. W. Contryman, J. Silcox, D. A. Stewart, G. Eda, C. Mattevi, S. Miller and M. Chhowalla, Atomic and electronic structure of graphene-oxide, Nano Lett., 2009, 9(3), 1058-1063.

10 T. Szabó, O. Berkesi, P. Forgó, K. Josepovits, Y. Sanakis, D. Petridis and I. Dékány, Evolution of surface functional groups in a series of progressively oxidized graphite oxides, Chem. Mater., 2006, 18(11), 2740-2749.

11 O. C. Compton and S. T. Nguyen, Graphene Oxide, Highly Reduced Graphene Oxide, and Graphene: Versatile Building Blocks for Carbon-Based Materials, Small, 2010, 6(6), 711-723.

12 B. Xu, S. Yue, Z. Sui, X. Zhang, S. Hou, G. Cao and Y. Yang, What is the choice for supercapacitors: graphene or graphene oxide?, Energy Environ. Sci., 2011, 4(8), 2826-2830.

13 S. H. Ha, Y. S. Jeong and Y. J. Lee, Free standing reduced graphene oxide film cathodes for lithium ion batteries, ACS Appl. Mater. Interfaces, 2013, 5(23), 12295-12303.

14 X. J. Hu, J. Hu, H. L. Dai, L. Ding and L. Jiang, Reduced graphene oxide and nanosheet-based nickel oxide microsphere composite as an anode material for lithium ion battery, Electrochim. Acta, 2012, 64, 23-28.

15 L. David, R. Bhandavat and G. Singh, $\mathrm{MoS}_{2} /$ graphene composite paper for sodium-ion battery electrodes, ACS Nano, 2014, 8(2), 1759-1770.

16 W. Gao, L. B. Alemany, L. Ci and P. M. Ajayan, New insights into the structure and reduction of graphite oxide, Nature Chem., 2009, 1(5), 403-408. 
17 J. A. Yan and M. Y. Chou, Oxidation functional groups on graphene: Structural and electronic properties, Phys. Rev. B: Condens. Matter Mater. Phys., 2010, 82(12), 125403.

18 B. Hammer and J. K. Nørskov, Electronic factors determining the reactivity of metal surfaces, Surf. Sci., 1995, 343(3), 211-220.

19 B. Hammer and J. K. Nørskov, Theoretical surface science and catalysis-calculations and concepts, Adv. Catal., 2000, 45, 71-129.

20 J. R. Kitchin, J. K. Nørskov, M. A. Barteau and J. G. Chen, Role of strain and ligand effects in the modification of the electronic and chemical properties of bimetallic surfaces, Phys. Rev. Lett., 2004, 93(15), 156801.

21 J. P. Perdew, K. Burke and M. Ernzerhof, Generalized gradient approximation made simple, Phys. Rev. Lett., 1996, 77(18), 3865-3868.

22 P. Giannozzi, S. Baroni, N. Bonini, M. Calandra, R. Car, C. Cavazzoni, D. Ceresoli, G. L. Chiarotti, M. Cococcioni, I. Dabo, A. D. Corso, S. Fabris, G. Fratesi, S. de Gironcoli, R. Gebauer, U. Gerstmann, C. Gougoussis, A. Kokalj, M. Lazzeri, L. Martin-Samos, N. Marzari, F. Mauri, R. Mazzarello, S. Paolini, A. Pasquarello, L. Paulatto, C. Sbraccia, S. Scandolo, G. Sclauzero, A. P. Seitsonen, A. Smogunov, P. Umari and R. M. Wentzcovitch, QUANTUM ESPRESSO: a modular and open-source software project for quantum simulations of materials, J. Phys.: Condens. Matter, 2009, 21(39), 395502.

23 Y. Tang, X. Dai, Z. Yang, L. Pan, W. Chen, D. Ma and Z. Lu, Formation and catalytic activity of Pt supported on oxidized graphene for the CO oxidation reaction, Phys. Chem. Chem. Phys., 2014, 16(17), 7887-7895.

24 A. S. Dobrota, I. A. Pašti and N. V. Skorodumova, Oxidized graphene as an electrode material for rechargeable metalion batteries-a DFT point of view, Electrochim. Acta, 2015, 176, 1092-1099.

25 H. J. Monkhorst and J. D. Pack, Special points for Brillouinzone integrations, Phys. Rev. B: Condens. Matter Mater. Phys, 1976, 13(12), 5188-5192.

26 R. F. W. Bader, Atoms in Molecules: A Quantum Theory, Clarendon, Oxford, UK, 1990.

27 G. Henkelman, A. Arnaldsson and H. Jónsson, A fast and robust algorithm for Bader decomposition of charge density, Comput. Mater. Sci., 2006, 36(3), 354-360.

28 S. Casolo, O. M. Lovvik, R. Martinazzo and G. F. Tantardini, Understanding adsorption of hydrogen atoms on graphene, J. Chem. Phys., 2009, 130(5), 054704.

29 P. A. Denis and F. Iribarne, On the hydrogen addition to graphene, J. Mol. Struct.: THEOCHEM, 2009, 907(1), 93-103.

30 S. C. Xu, S. Irle, D. G. Musaev and M. C. Lin, Quantum chemical study of the dissociative adsorption of $\mathrm{OH}$ and $\mathrm{H}_{2} \mathrm{O}$ on pristine and defective graphite (0001) surfaces: reaction mechanisms and kinetics, J. Phys. Chem. C, 2007, 111(3), 1355-1365.

31 N. Ghaderi and M. Peressi, First-principle study of hydroxyl functional groups on pristine, defected graphene, and graphene epoxide, J. Phys. Chem. C, 2010, 114(49), 21625-21630.

32 R. J. W. E. Lahaye, H. K. Jeong, C. Y. Park and Y. H. Lee, Density functional theory study of graphite oxide for different oxidation levels, Phys. Rev. B: Condens. Matter Mater. Phys., 2009, 79(12), 125435.

33 T. O. Wehling, M. I. Katsnelson and A. I. Lichtenstein, Impurities on graphene: Midgap states and migration barriers, Phys. Rev. B: Condens. Matter Mater. Phys., 2009, 80(8), 085428.

34 M. Yang, L. Zhou, J. Wang, Z. Liu and Z. Liu, Evolutionary chlorination of graphene: from charge-transfer complex to covalent bonding and nonbonding, J. Phys. Chem. C, 2011, 116(1), 844-850.

35 I. Fampiou and A. Ramasubramaniam, Binding of Pt nanoclusters to point defects in graphene: adsorption, morphology, and electronic structure, J. Phys. Chem. C, 2012, 116(11), 6543-6555.

36 Z. Jovanović, I. Pašti, A. Kalijadis, S. Jovanović and Z. Laušević, Platinum-mediated healing of defective graphene produced by irradiating glassy carbon with a hydrogen ion-beam, Mater. Chem. Phys., 2013, 141(1), 27-34.

37 A. Bagri, C. Mattevi, M. Acik, Y. J. Chabal, M. Chhowalla and V. B. Shenoy, Structural evolution during the reduction of chemically derived graphene oxide, Nature Chem., 2010, 2(7), 581-587.

38 Y. S. Puzyrev, B. Wang, E. X. Zhang, C. X. Zhang, A. K. M. Newaz, K. I. Bolotin, D. M. Fleetwood, R. D. Schrimpf and S. T. Pantelides, Surface reactions and defect formation in irradiated graphene devices, IEEE Trans. Nucl. Sci., 2012, 59(6), 3039-3044.

39 W. Humphrey, A. Dalke and K. Schulten, VMD: visual molecular dynamics, J. Mol. Graphics, 1996, 14(1), 33-38.

40 D. Vasić, Z. Ristanović, I. Pašti and S. Mentus, Systematic DFTGGA study of hydrogen adsorption on transition metals, Russ. J. Phys. Chem. A, 2011, 85(13), 2373-2379.

41 K. Spyrou, D. Gournis and P. Rudolf, Hydrogen storage in graphene-based materials: efforts towards enhanced hydrogen absorption, ECS J. Solid State Sci. Technol., 2013, 2(10), M3160-M3169.

42 H. Sahin and S. Ciraci, Chlorine adsorption on graphene: Chlorographene, J. Phys. Chem. C, 2012, 116(45), 24075-24083.

43 F. Karlicky, K. Kumara Ramanatha Datta, M. Otyepka and R. Zbořil, Halogenated graphenes: rapidly growing family of graphene derivatives, ACS Nano, 2013, 7(8), 6434-6464.

44 F. Rodríguez-Reinoso, The role of carbon materials in heterogeneous catalysis, Carbon, 1998, 36(3), 159-175.

45 J. L. Figueiredo, M. F. R. Pereira, M. M. A. Freitas and J. J. M. Orfao, Modification of the surface chemistry of activated carbons, Carbon, 1999, 37(9), 1379-1389.

46 Y. R. Tzeng, W. W. Pai, C. S. Tsao and M. S. Yu, Adsorption of single platinum atom on the graphene oxide: the role of the carbon lattice, J. Phys. Chem. C, 2011, 115(24), 12023-12032.

47 F. Abild-Pedersen, J. Greeley, F. Studt, J. Rossmeisl, T. R. Munter, P. G. Moses, E. Skúlason, T. Bligaard and J. K. Nørskov, Scaling properties of adsorption energies for hydrogen-containing molecules on transition-metal surfaces, Phys. Rev. Lett., 2007, 99(1), 016105.

48 A. Kokalj, XCrySDen - a new program for displaying crystalline structures and electron densities, J. Mol. Graphics Modell., 1999, 17(3), 176-179.

49 V. Tozzini and V. Pellegrini, Reversible hydrogen storage by controlled buckling of graphene layers, J. Phys. Chem. C, 2011, 115(51), 25523-25528. 